\title{
Effect of Plant Growth Regulators, Organic, Inorganic and Biofertilizer on Seed Yield and Vigour Characters in Green Gram (Vigna radiata $\mathrm{L}$. wilczek)
}

\author{
Sudheer Pathak ${ }^{1 *}$, Neha Srivastava ${ }^{1}$ and G. R. Lavanya ${ }^{2}$ \\ ${ }^{1}$ Department of Genetics and Plant Breeding, School of Agriculture, ITM University, \\ Gwalior-M.P, 474001 \\ ${ }^{2}$ Department of Genetics and Plant Breeding, Naini Agricultural Institute, \\ Prayagraj, U.P, 211007, India \\ *Corresponding author
}

\section{A B S T R A C T}

Keywords

Gibberelic acid $\left(\mathrm{GA}_{3}\right)$, Biofertilizer, Organic fertilizer, Inorganic fertilizer, Greengram, Seed vigour

Article Info

Accepted:

12 December 2020

Available Online:

10 January 2021
The present investigation comprised of 13 treatments including control carried out in field experimentation, centre of the Department of Genetics and Plant Breeding, Sam Higginbottom University of Agriculture, Technology and Sciences, Prayagraj during kharif 2012. The experiment was conducted to study the effect of plant growth regulators, organic, inorganic and biofertilizer on seed yield and seed vigour characters in greengram. The data were recorded on 18 characters. Based on the mean performance the treatment $\mathrm{T}_{1}(50 \% \mathrm{RDN}+\mathrm{FYM} @ 2$ tones per ha + Rhizobium $20 \mathrm{~g}$ per kg seed $+\mathrm{GA}_{3}$ @ $40 \mathrm{PPM}$ ) was identified as best treatment for seed yield. $\mathrm{T}_{1}$ obtained high germination $(\%)$, root length $(\mathrm{cm})$, shoot length $(\mathrm{cm})$, seedling length $(\mathrm{cm})$, seedling vigour index, seedling dry weight $(\mathrm{mg})$ and seed yield. Interaction effect of variety with plant growth regulator and biofertilizer was significant for all the characters except plant height and seedling dry weight (mg). Thus it indicate that plant growth regulator with biofertilizer may be better to achieve higher seed yield and yield components in greengram.

\section{Introduction}

Greengram is one of the most ancient and extensively grown leguminous crops of India. In mungbean seed, there is $51 \%$ carbohydrate, $26 \%$ protein, $3 \%$ minerals and $3 \%$ vitamins. In India it occupies 3.0 million ha. area with a production of 1.24 million tonnes with the average yield $425 \mathrm{~kg}$ per ha. (Anon., 2009a).
The factor attributes for low yield of pulses in India as compared to the world productivity are non-availability of quality seeds of improved and short duration varieties, growing of pulses under marginal and less fertile soil with low inputs and without pest and disease management. So, we can improve the production potential of this crop by use of plant growth regulators, organic, inorganic 
and biofertilizers. Plant hormones in a broad sense are organic compounds which play an important role in plant growth development and yield of crops to prevent the fruit and flower drop for a longer period. The application of GA to seeds can bypass the dormancy requirement and speed up the germination process. Bio-fertilizers being essential components of organic farming play vital role in maintaining long term soil fertility and sustainability by fixing atmospheric dinitrogen $(\mathrm{N}=\mathrm{N})$, mobilizing fixed macro and micro nutrients or convert insoluble $\mathrm{P}$ in the soil into forms available to plants, there by increases their efficiency and availability (Venkatashwarlu, 2008a). Rhizobium fix nitrogen 50-100 kg/ha. with legumes only (Wani and Lee 2002). Hence, there is a scope for improving the production potential of this crop by use of plant growth regulators, organic, inorganic and biofertilizers.

\section{Materials and Methods}

The experimental material comprised of 13 treatment on greengram. The experiment was conducted at the field experimentation centre and under laboratory condition of Department of Genetics and Plant Breeding, Sam Higginbottom University of Agriculture, Technology and Sciences, Prayagraj. Each treatment was grown in a plot of $3^{2} \mathrm{~m}$ spaced at $30 \times 10 \mathrm{~cm}$ apart. Standard agronomic practices compatible with this ago-ecological zone were adopted to ensure good crop growth.

\section{Data collection}

Observations were recorded on 5 randomly selected plants from each replication for various characters viz., days to $50 \%$ flowering, Number of branches per Plant, Number of leaves per Plant, Number of cluster per Plant, Number of pods per Plant,
Pod weight per Plant, Number of seed per pod, Number of seed per Plant, Plant height, Days to Maturity, Seed index, Seed yield per Plant, Germination percentage, Root length, shoot length, seedling length, seedling vigour index and seedling dry weight.

Statistical analysis: The data were calculated by the analysis of variance and critical difference. The analysis of variance was worked to test the significance of F- tests as per the methodology suggested by FISHER (1936).

\section{Results and Discussion}

The analysis variance for different characters was presented in table 4.1. Analysis of variance revealed significant differences among 13 treatments for all the characters except plant height, seed index and days to maturity.

Analysis of variance for seed vigour characters were furnished in table 4.2. All treatments significant differed for all characters studied except seedling dry weight. Similar findings were also observed in earlier studies (Bhat et al., 2009 and Suty 1984).

Based on the mean performance table (Table 4.3) treatment $\mathrm{T}_{1}(50 \% \mathrm{RDN}+\mathrm{FYM} @ 2$ tones per ha + Rhizobium $20 \mathrm{~g}$ per $\mathrm{kg}$ seed + $\mathrm{GA}_{3} @ 40$ PPM) was identified as best treatment for seed yield. Similar findings were also observed in earlier studies (Bai et al., 1987 and Prasad and Ram 1992). $\mathrm{T}_{1}$ obtained high germination percentage (\%), root length $(\mathrm{cm})$, shoot length $(\mathrm{cm})$, seedling length $(\mathrm{cm})$, seedling vigour index and seedling dry weight $(\mathrm{mg})$. The increase in germination percentage may be due to a better metabolic response of the seed to application of plant growth regulators $\left(\mathrm{GA}_{3}, \mathrm{IAA}\right)$. Naidu (2001) reported that the importance of IAA was indicated with respect to seed 
germination process but Gibberellic acid was found to be more effective in improving seed germination than either IBA or IAA. Interaction effect of variety with plant growth regulator biofertilizer was maximum significant for all characters. This indicate that plant growth regulator with biofertilizer may be better to achieve higher seed yield and yield components in greengram.

It is concluded that different treatments showed significantly different effects on all yield characters and also on seed vigour characters studied except plant height, days to maturity, seed index and seedling dry weight on greengram cv Samrat. Greengram after treating with 50\% RDN + FYM @ 2 tones per ha + Rhizobium $20 \mathrm{~g}$ per kg seed $+\mathrm{GA}_{3} @ 40$ PPM $\left(\mathrm{T}_{1}\right)$ recorded high seed yield than other treatments. Further experimentation is suggested for the confirmation of obtained results.

Table.1 Analysis of variance for 12 yield characters in greengram

\begin{tabular}{|l|l|c|c|c|}
\hline \multirow{2}{*}{ S.No. } & Characters & \multicolumn{3}{|c|}{} \\
\cline { 3 - 5 } & & $\begin{array}{c}\text { Replication } \\
(\mathbf{d} \mathbf{f}=\mathbf{0 2})\end{array}$ & $\begin{array}{c}\text { Treatments } \\
(\mathbf{d} \mathbf{f}=\mathbf{1 2})\end{array}$ & $\begin{array}{c}\text { Error } \\
(\mathbf{d} \mathbf{f}=\mathbf{2 4})\end{array}$ \\
\hline $\mathbf{1}$ & Plant height $(\mathrm{cm})$ & 33.07 & 24.37 & $\mathbf{1 5 . 9 2}$ \\
\hline $\mathbf{2}$ & Number of branches per plant & 0.20 & $0.60^{* *}$ & $\mathbf{0 . 2 2}$ \\
\hline $\mathbf{3}$ & Number of leaves per plant & 0.82 & $5.75^{* *}$ & $\mathbf{1 . 3 3}$ \\
\hline $\mathbf{4}$ & Days to 50\% flowering & 0.18 & $6.56^{* *}$ & $\mathbf{0 . 8 5}$ \\
\hline $\mathbf{5}$ & Days to maturity & 1.26 & 2.08 & $\mathbf{1 . 9 8}$ \\
\hline $\mathbf{6}$ & Number of pods per plant & 1.65 & $8.62^{* *}$ & $\mathbf{0 . 7 1}$ \\
\hline $\mathbf{7}$ & Pod weight per plant & 0.66 & $10.34^{* *}$ & $\mathbf{0 . 8 1}$ \\
\hline $\mathbf{8}$ & Number of seeds per pod & 0.07 & $2.20^{* *}$ & $\mathbf{0 . 2 3}$ \\
\hline $\mathbf{9}$ & Number of seeds per plant & 358.11 & $822.68^{* *}$ & $\mathbf{2 2 0 . 0 7}$ \\
\hline $\mathbf{1 0}$ & Seed index (\%) & 0.09 & 0.24 & $\mathbf{0 . 2 7}$ \\
\hline $\mathbf{1 1}$ & Number of clusters per plant & 0.31 & $1.38^{* *}$ & $\mathbf{0 . 0 5}$ \\
\hline $\mathbf{1 2}$ & Seed yield per plant & $\mathbf{0 . 1 5}$ & $\mathbf{2 . 0 2} * *$ & $\mathbf{0 . 7 2}$ \\
\hline
\end{tabular}

$* *$ significant at $1 \%$ level of significance.

Table.2 Analysis of variance for seed vigour characters in greengram

\begin{tabular}{|c|l|c|c|}
\hline \multirow{2}{*}{ S.No } & \multicolumn{1}{|c|}{ Characters } & \multicolumn{2}{|c|}{ Mean sum of sq. } \\
\cline { 3 - 4 } & & $\begin{array}{c}\text { Treatments } \\
(\mathbf{d . f} \mathbf{f}=\mathbf{1 2})\end{array}$ & $\begin{array}{c}\text { Error } \\
(\mathbf{d} . \mathbf{f}=\mathbf{2 4})\end{array}$ \\
\hline $\mathbf{1}$ & Germination $\%$ & $75.65^{* *}$ & $\mathbf{1 . 1 1}$ \\
\hline $\mathbf{2}$ & Root length $(\mathrm{cm})$ & $1.25^{* *}$ & $\mathbf{0 . 0 1}$ \\
\hline $\mathbf{3}$ & Shoot length $(\mathrm{cm})$ & $12.22^{* *}$ & $\mathbf{0 . 3 5}$ \\
\hline $\mathbf{4}$ & Seedling length $(\mathrm{cm})$ & $21.25^{* *}$ & $\mathbf{0 . 3 9}$ \\
\hline $\mathbf{5}$ & Seedling vigour index & $253837.83^{* *}$ & $\mathbf{1 6 9 6 . 1 9}$ \\
\hline $\mathbf{6}$ & Seedling dry weight $(\mathbf{m g})$ & $\mathbf{0 . 0 2}$ & $\mathbf{0 . 0 1}$ \\
\hline
\end{tabular}

** significant at $1 \%$ level of significance 
Int.J.Curr.Microbiol.App.Sci (2021) 10(01): 1947-1951

Table.3 Mean performance of greengram after seed treatment for seed yield and yield characters

\begin{tabular}{|c|c|c|c|c|c|c|c|c|c|c|c|c|c|}
\hline S.No. & Treatment & $\begin{array}{l}\text { Plant } \\
\text { height }\end{array}$ & $\begin{array}{c}\text { No. of } \\
\text { branches/p } \\
\text { lant }\end{array}$ & $\begin{array}{c}\text { No. of } \\
\text { leaves/pl } \\
\text { ant }\end{array}$ & $\begin{array}{c}\text { Days to } \\
50 \% \\
\text { flowering }\end{array}$ & $\begin{array}{l}\text { No. of } \\
\text { clusters }\end{array}$ & $\begin{array}{l}\text { No. of } \\
\text { pods/ } \\
\text { plant }\end{array}$ & $\begin{array}{c}\text { Pod } \\
\text { weight/ } \\
\text { plant }\end{array}$ & $\begin{array}{c}\text { No. of } \\
\text { seed/pod }\end{array}$ & $\begin{array}{c}\text { No.of } \\
\text { seed/pla } \\
\text { nt }\end{array}$ & $\begin{array}{c}\text { Days to } \\
\text { maturity }\end{array}$ & $\begin{array}{c}\text { Seed } \\
\text { index }(\%)\end{array}$ & $\begin{array}{c}\text { Seed } \\
\text { yield/p } \\
\text { lant }\end{array}$ \\
\hline 1 & $\mathrm{~T} 1$ & 31.80 & 6.67 & 19.40 & 38.33 & 6.53 & 22.20 & 22.38 & 12.13 & 192.00 & 54.00 & 4.77 & 8.10 \\
\hline 2 & $\mathrm{~T} 2$ & 27.67 & 6.93 & 20.70 & 36.33 & 5.60 & 18.80 & 18.41 & 9.20 & 155.00 & 53.00 & 4.03 & 6.33 \\
\hline 3 & T3 & 34.00 & 6.93 & 20.80 & 36.00 & 5.33 & 18.27 & 17.88 & 9.47 & 159.33 & 51.67 & 3.87 & 5.50 \\
\hline 4 & $\mathrm{~T} 4$ & 34.60 & 7.23 & 21.71 & 37.00 & 6.07 & 21.73 & 21.73 & 10.33 & 188.07 & 52.33 & 4.00 & 7.03 \\
\hline 5 & T5 & 33.33 & 6.90 & 20.80 & 34.67 & 5.47 & 18.20 & 17.80 & 8.80 & 168.33 & 51.33 & 4.27 & 6.60 \\
\hline 6 & T6 & 32.97 & 6.83 & 20.50 & 38.67 & 5.27 & 19.00 & 18.69 & 9.40 & 155.00 & 53.00 & 4.00 & 5.90 \\
\hline 7 & $\mathrm{~T} 7$ & 35.67 & 7.60 & 22.80 & 37.00 & 6.20 & 21.00 & 21.09 & 10.07 & 178.67 & 53.67 & 4.43 & 6.27 \\
\hline 8 & T8 & 35.57 & 7.40 & 21.20 & 36.67 & 4.87 & 17.23 & 16.71 & 9.53 & 160.40 & 52.33 & 4.20 & 6.17 \\
\hline 9 & T9 & 38.70 & 8.27 & 24.80 & 37.67 & 5.13 & 17.93 & 18.18 & 9.53 & 165.40 & 52.67 & 4.00 & 6.37 \\
\hline 11 & $\mathrm{~T} 11$ & 29.60 & 6.70 & 20.20 & 34.33 & 5.13 & 19.47 & 17.60 & 9.40 & 161.60 & 53.00 & 4.27 & 6.30 \\
\hline 12 & $\mathrm{~T} 12$ & 35.33 & 7.13 & 20.63 & 34.67 & 5.13 & 17.67 & 17.48 & 9.20 & 142.80 & 53.33 & 4.40 & 5.87 \\
\hline 13 & $\mathrm{~T} 13$ & 35.63 & 7.37 & 22.10 & 34.33 & 3.93 & 17.73 & 17.00 & 8.87 & 136.67 & 52.67 & 4.23 & 4.97 \\
\hline \multicolumn{2}{|c|}{ Mean } & 33.69 & 7.13 & 21.22 & 36.25 & 5.44 & 19.25 & 18.84 & 9.73 & 164.98 & 52.64 & 4.20 & 6.38 \\
\hline \multirow[t]{3}{*}{ Range } & Max. & 38.70 & 7.60 & 24.80 & 38.67 & 6.53 & 22.20 & 22.38 & 12.13 & 192.00 & 54.00 & 4.77 & 8.10 \\
\hline & Min. & 27.67 & 6.67 & 19.40 & 34.33 & 3.93 & 17.67 & 16.71 & 8.87 & 136.67 & 51.33 & 3.87 & 4.97 \\
\hline & $\mathrm{CD}$ & 6.72 & 0.79 & 1.94 & 1.94 & 0.39 & 0.39 & 1.52 & 0.18 & 25 & 2.37 & 0.87 & 1.43 \\
\hline
\end{tabular}


Table.4 Mean performance table of seed vigour parameter in greengram

\begin{tabular}{|c|c|c|c|c|c|c|}
\hline Treatment & Germination \% & $\begin{array}{l}\text { Root length } \\
\text { (cm) }\end{array}$ & $\begin{array}{l}\text { Shoot length } \\
(\mathrm{cm})\end{array}$ & $\begin{array}{l}\text { Seedling length } \\
(\mathbf{c m})\end{array}$ & $\begin{array}{c}\text { Seedling } \\
\text { vigour index }\end{array}$ & $\begin{array}{c}\text { Seedling dry } \\
\text { weight(mg) }\end{array}$ \\
\hline T1 & $94.00 *$ & $5.32 *$ & $14.12 *$ & $19.44 *$ & $1827.44 *$ & $0.16^{*}$ \\
\hline T2 & 72.50 & 3.83 & 7.77 & 11.60 & 840.95 & 0.11 \\
\hline T3 & 80.00 & 4.81 & 11.81 & 16.42 & 1314.54 & 0.13 \\
\hline T4 & $91.00 *$ & $5.11^{*}$ & $13.13^{*}$ & $18.24 *$ & $1695.39 *$ & $0.14^{*}$ \\
\hline T5 & 75.50 & 3.84 & 8.19 & 12.03 & 908.12 & 0.11 \\
\hline T6 & $83.00 *$ & $4.94 *$ & 11.66 & 16.60 & $1495.66^{*}$ & 0.12 \\
\hline T7 & $85.50 *$ & $5.01 *$ & $13.16^{*}$ & $18.49 *$ & $1580.63^{*}$ & $0.14^{*}$ \\
\hline T8 & 78.50 & 3.11 & 7.57 & 10.68 & 838.39 & 0.11 \\
\hline T9 & 82.00 & $4.95 *$ & 11.95 & 16.90 & 1385.84 & 0.12 \\
\hline T10 & $86.50 *$ & $4.99 *$ & $12.96^{*}$ & $17.95 *$ & $1552.74 *$ & 0.13 \\
\hline T11 & 75.50 & 2.91 & 6.78 & 9.69 & 731.65 & 0.12 \\
\hline T12 & 80.50 & 4.78 & 11.60 & 16.37 & 1317.86 & 0.13 \\
\hline T13 control) & 80.50 & 4.69 & 11.57 & 16.26 & 1309.06 & 0.12 \\
\hline SE & 1.05 & 0.01 & 0.35 & 0.39 & 1696.19 & 0.01 \\
\hline CD at $5 \%$ & 2.28 & 0.16 & 1.28 & 1.35 & 88.96 & 0.02 \\
\hline
\end{tabular}

* Significant at $1 \%$ level

\section{References}

Anonymous. 2004. Recommended dietary allowance for Indians. Survey of Indian Agriculture, Pub. The Hindu. pp54.

Anonymous. 2009. Agriculture Centre for monitoring Indian Economy, Economic Intelligence Service, Andheri (East), Mumbai,

Bai D.I.S., Abraham A.T. and Mercy S.T. 1987. Hormonal influence of crop performance in green gram. Legume Res., 10(1): 49-52.

Bhat M.I., Rashid A., Rasool F., Mahdi S.S., Haq S.A. and Bhat R.A. 2010. Effect of Rhizobium and VA-mycorrhizae on greengram under temperate conditions. Res. J. of Agric. Sci., 1(2): 113-116.

Fisher R.A. 1936. The correlations between relative on the supposition of genotype grown in Kumaun Himalaya, Indian $J$. Genet., 66(1): 37-38.

Naidu C.V. 2001. Improvement of seed germination in red sanders (Pterocarpus santalinus Linn. F) By plant growth regulators. Indian J. Plant. Physiol., 6: 205-207.

Prasad J. and H. Ram. 1992. Effect of Zinc, Copper and and Rhizobium inoculation on microbial population of soil and yield of greengram ((Vigna radiata). Intl. J. Tropic. Agric., 10(2): 157-160.

Suty L. 1984. Growth regulator and potential of faba bean. Cultivar., 171: 71-73.

Venkatashwarlu B. 2008. Role of bio-fertilizers in organic farming: Organic farming in rain fed agriculture: Central institute for dry land agriculture, Hyderabad, pp 85-95.

Wani S.P. and Lee K.K. 2002. Population dynamics of nitrogen fixing bacteria associated with pearl millet $(P$. americanum L.). In biotechnology of nitrogen fixation in the tropics. University of Pertanian, Malaysia, pp 21-30.

\section{How to cite this article:}

Sudheer Pathak, Neha Srivastava and Lavanya, G. R. 2021. Effect of Plant Growth Regulators, Organic, Inorganic and Biofertilizer on Seed Yield and Vigour Characters in Green Gram (Vigna radiata L. wilczek). Int.J.Curr.Microbiol.App.Sci. 10(01): 1947-1951. doi: https://doi.org/10.20546/ijcmas.2021.1001.226 